\title{
Topographical relocation of adolescent sleep spindles reveals a new maturational pattern in the human brain
}

Ferenc Gombos ${ }^{\mathrm{a}, \mathrm{b}}$, Róbert Bódizs ${ }^{\mathrm{c}, \mathrm{d}}$, Adrián Pótári ${ }^{\mathrm{b}}$, Gábor Bocskai ${ }^{\mathrm{a}}$, Andrea Berencsi ${ }^{\mathrm{b}}$, e, Hanna Szakács ${ }^{\text {a }}$ and Ilona Kovács a, b, f, *

${ }^{a}$ Laboratory for Psychological Research, Pázmány Péter Catholic University, Budapest, 1088 Hungary

b Adolescent Development Research Group, Hungarian Academy of Sciences - Pázmány Péter Catholic University, Budapest, 1088 Hungary

${ }^{c}$ Institute of Behavioural Sciences, Semmelweis University, Budapest, 1089 Hungary

${ }^{\mathrm{d}}$ National Institute of Clinical Neurosciences, Budapest, 1145 Hungary

${ }^{\mathrm{e}}$ Institute for the Methodology of Special Needs Education and Rehabilitation, Bárczi Gusztáv Faculty of Special Needs Education, Eötvös Loránd University, Budapest, 1097 Hungary

${ }^{\mathrm{f}}$ Institute of Cognitive Neuroscience and Psychology, Research Centre for Natural Sciences, Budapest, 1117 Hungary

*corresponding author: Ilona Kovács, Laboratory for Psychological Research, Pázmány Péter Catholic University, 1 Mikszáth Kálmán sq., Budapest, 1088 Hungary

Email: dr.ilona.kovacs@gmail.com

Author Contributions: IK, FG and RB designed the experiments; FG, AP and GB analysed the data; all authors contributed to writing the paper.

Competing Interest Statement: none

Classification: Biological Sciences, Neuroscience

Keywords: sleep spindles, adolescent brain, cortical maturation, precuneus

\section{This PDF file includes:}

Main Text

Figures 1 to 3

Supporting Information 


\begin{abstract}
Current theories of human neural development emphasize the posterior-to-anterior (backto-front) pattern of brain maturation as an unequivocal ontogenetic feature that is largely due to the late maturation of the frontal lobes. However, this unidirectional developmental scenario does not take into account those areas of the brain that are not directly involved with sensory input and behavioral control. We suggest that the investigation of spontaneous cortical activity in the absence of sensory stimulation, such as sleep, might help to build a more complete account of the developing brain. Here we investigate the adolescent transformations in the topography of sleep spindles. Sleep spindles are known to be involved in neural plasticity, and in the adult, have a bimodal topography: slow spindles are frontally dominant, while fast spindles have a parietal/precuneal origin. The late functional segregation of the precuneus from the frontoparietal network during adolescence suggests that spindle topography might approach the adult state relatively late in development, and it may not be a result of the back-to-front maturational pattern. We analyzed the topographical distribution of spindle parameters in HD-EEG polysomnographic sleep recordings of 60 adolescent and young adult participants and found that slow spindle duration maxima are traveling from central to anterior brain regions, while fast spindle density, amplitude and frequency peaks are traveling from central to more posterior brain regions. These results provide evidence for the gradual posteriorization of the predominant anatomical localization of fast sleep spindles during adolescence and indicate the existence of a front-to-back pattern of human brain maturation.

\section{Significance statement}

The human brain has an exceptionally long maturational course, and we are only beginning to understand the detailed pattern of structural and functional changes taking place after puberty. We investigate the adolescent transformations in the topography of sleep spindles in order to reveal maturational patterns of the large-scale functional networks of the human brain. Sleep spindles are known to be involved in neural plasticity, and in the adult, have a bimodal topography: slow spindles are frontally dominant, while fast spindles have a parietal/precuneal origin. According to our findings, slow spindle parameters show a central to frontal maturation, while fast spindle parameters mature in a central to posterior direction. These results reveal a novel front-to-back pattern of human brain maturation.
\end{abstract}




\section{Main Text}

\section{Introduction}

The structural and functional development of the human brain has an exceptionally extended temporal window as compared to other vertebrates (1-3). This extension provides the developing adolescent human brain with an astonishing learning capacity for the final groundwork of adult life, and it is also a period of marked vulnerability (4). It is of utmost relevance to describe the adolescent patterns of brain maturation in detail if we hope to see the next generation to grow into healthy and talented adults.

Details on the protracted maturational course of the human brain have been accumulating with the increasingly advanced brain imaging technologies (5-7). Up to date, there are two relevant patterns described in terms of the process of reaching structural maturity: one is the back-to-front propagation of brain maturation, with the frontal areas achieving adult proportions the latest (6-8), and the other is the dual-systems model where the ventral affective system precedes the maturation of the prefrontal control system (9-11). While these are generally accepted patterns of brain maturation, we would like to add a further, functionally relevant and supposedly human-specific maturational arrangement to this scenario.

According to the latest accounts of adolescent functional connectivity it seems that the association areas or the so-called default mode network (DMN) of the brain are remodeled during development at a later age than those areas that are more related to immediate sensory input and behavioral control $(12,13)$. While the latest maturing hot-spots in the network subserving "internal" cognition are believed to be fundamental to human evolution (14-16), their exact connectivity and contribution to adolescent development is not yet known. Here we suggest that the investigation of spontaneous cortical activity in the absence of sensory stimulation, such as sleep, might help this field move forward, and a more complete account of the developing large-scale functional networks will emerge.

The dynamics of large-scale brain networks in humans are tightly linked with neural oscillatory activities, the latter reflecting underlying functional connectivity, as well as contributing to information coding and integration in the neural system. Sleep spindle oscillations are groups of 11-16 Hz waves emerging in non-rapid eye movement (NREM) sleep as a result of the rhythmic hyperpolarization rebound sequences of thalamocortical neurons, the inhibition being initiated in the thalamic reticular nucleus (17). Sleep spindles are hypothesized to reflect the underlying large-scale cortical functional networks emerging from spontaneous neural activity during sleep $(18,19)$. A bimodal frequency and topography of sleep spindles has been described in adults. That is, slow $(\sim 12 \mathrm{~Hz})$ sleep spindles are frontally dominant, whereas fast $(\sim 14 \mathrm{~Hz})$ sleep spindles are of parietal/precuneal origin in adult humans $(20,21)$. 
The precuneus is a major hub of the DMN, part of the complex neurocognitive network involved in the maintenance of conscious awareness, self-reflection, visuospatial integration and episodic memory $(16,22)$. Similar to the frontal cortex, the precuneus expansion is a neurological specialization of Homo sapiens and is assumed to be associated with recent human cognitive specializations (23). In addition, the precuneus has been characterized by an increasing functional segregation from the frontoparietal network between 8 and 26 years of age (24). Thus, besides of the late maturation in frontal lobe functions, characterized by the well-known back-to-front maturation pattern of the brain $(6-8,25)$, a more posterior neuroanatomical structure, namely the precuneus is subject of significant developmental changes in pubertal and adolescent ages as well.

Based on the relatively late functional segregation of the precuneus from the frontoparietal network, as well as on the bimodal prefrontal versus precuneal sources of slow and fast sleep spindles, respectively, we postulate that these two sleep spindle types undergo a topographical transformation characterized by increasing anatomical segregation during adolescent development. We assume that slow and fast sleep spindles are gradually relocated from the more central toward the prefrontal and the parietal regions, respectively. The presumed age-related topographical transfer is supposed to reflect the increasing differentiation and segregation of the frontal and precuneal subsystems in the DMN, as well as the associated late development of the phylogenetically new neuroanatomical structures in humans.

Here we focus on the late maturation of individually adjusted slow-frontal and fast-parietal sleep spindle features in adolescence by using HD-EEG recordings in three subgroups of different ages $(12,16$ and 20 years). We hypothesize that the two sleep spindle types index distinct neurodevelopmental processes during the course of adolescent brain maturation and functional specialization. We rely on HD-EEG recordings in order to increase spatial resolution relative to classical EEG studies. We believe that focusing on the bimodal antero-posterior source and frequency distribution of sleep spindle oscillations $(20,21)$, contrasting the unimodal, largely frontal maxima of slow waves $(26,27)$ provides us with a better understanding of the adolescent remodeling of the human brain.

\section{Results}

60 adolescent and young adult subjects participated in the study $(12,16$ and 20 years of age, $n=20$ in each age group with a perfect gender balance, see Materials and Methods/Participants). We recorded 128 channel full-night sleep HD-EEG data and detected individual-specific slow and fast spindles by the Individual Adjustment Method (IAM, see Materials and Methods/Procedure and ref. (28)). We analyzed the derivationspecific topographical distribution of the spindle parameters and their age-dependent differences, and calculated average spindle density, duration, maximum amplitude and peak frequency parameters for NREM sleep. Peak frequency detection relied on the center of gravity $(\mathrm{CoG})$ of the individualized spectral peak frequencies in the spindle range (11- 
$16 \mathrm{~Hz}$ ). General Linear Model (GLM) was implemented on averaged slow spindle measures of the frontopolar and frontal region (FPF) as well as on averaged fast spindle measures of the centro-parietal (CP) region. We tested the null hypothesis at the descriptive significance of $\mathrm{p}<.05$ and performed post hoc test Fisher's LSD. The distribution of the spindle parameters along the midline derivations (from Fpz to Iz) was calculated, and the maximum position of the distribution for every spindle parameter was determined. We scrutinized anterior-posterior shifts of the maximum locations as a function of age.

Here we first describe age- and sex-related spindle parameters with illustrations and a detailed analysis provided in the Supplementary Information, then we portray the topographical distribution and age-related topographical changes of slow and fast spindle parameters (Figures 1 and 2). Finally, evidence for age-related antero-posterior shifts in sleep spindle parameters is presented (Figure 3).

Slow and fast spindle densities display slight but non-significant decline with age. Spindle duration and amplitude decline significantly for both spindle types as a function of age (for statistical details, please consult SI Figurela-c). Slow spindle frequency increases significantly between 16 and 20 years of age, while fast spindle frequency starts to increase already at 12 and goes through a significant change up to the age of 20 (see SI Figure 1d). There was no evidence for the influence of sex on the development of NREM sleep spindle parameters except for frequency, where we found that 20-year-old females have significantly higher spindle frequencies for both spindle types, and for slow spindles this effect is observed in 12-year-olds as well (see SI Figure 2 for statistical details).

\section{Topographical distribution and age-related topographical changes of slow and fast spindle parameters}

The age-dependent topographical distributions of slow and fast spindle parameters are shown in Figures 1 and 2, respectively.

In terms of duration, decrease in slow spindles from age 12 to 16 was Rüger area significant for all recording locations $(\mathrm{T}=(-2.05--3.29), \mathrm{p}=(.002-.04))$. Maximum amplitude values of slow spindles decreased with frontal and frontopolar foci between all age bins from 12 to 20 years $(\mathrm{T}=(-2.03--2.99),(\mathrm{p}<.001-.048))$. Frequency increased Rüger area significantly at all derivations with a frontal focus throughout the entire adolescent period $(\mathrm{T}=(3.2-3.28), \mathrm{p}=(.002-.003))$, see Figure 1 .

Fast spindle density increased Rüger area significantly at the perimeter of the scalp ( $\mathrm{T}=(-$ $2.07--3.06), \mathrm{p}=(.04-.004))$. Fast spindles revealed a significant decrease in duration for all recording derivations over the entire adolescent period from age 12 to 20 ( $\mathrm{T}=(-2.06-$ $4.54),(\mathrm{p}<.001-.047))$. Fast spindle maximum amplitude values decreased significantly in all areas with a frontal and central focus $(\mathrm{T}=(-2.05--4.54),(\mathrm{p}<.001-.048))$. Fast spindle 
frequency values monotonously increased with age. The differences are Rüger area significant for the entire cortex in all age-bins $(\mathrm{T}=(5.37-5.46), \mathrm{p}<.001)$, see Figure 2.

\section{Place Figures 1 and 2 about here}

\section{Age-related shifts in sleep spindle parameters}

Topographical shifts of slow and fast spindle parameters can be visually assessed in the topographical distribution maps of Figure 1 and 2, respectively. While slow spindle parameters tended to shift towards anterior, fast sleep spindle measures seemed to shift towards posterior regions with age. In order to more precisely quantify these observable tendencies, we calculated the distribution of spindle parameters along the midline derivations (from Fpz to Iz) and determined the maximum position of the distribution for every spindle parameter.

Maximum values of slow spindle duration shifted towards frontal regions. The difference of the positions between the age groups of 12 and 16 years of age was statistically significant $(p<.05)$. In terms of fast spindle density and frequency, the difference of positions of maximum values was significant in the age groups of 12 to $16(\mathrm{p}<.01)$ and 12 to $20(p<.001)$, revealing a central to centro-parietal shift with age. Maximum values of the positions of fast spindle maximum amplitudes shifted significantly from central to centroparietal regions in the age groups of 16 to $20(\mathrm{p}<.01)$ and 12 to $20(\mathrm{p}<.001)$, but not between ages 12 to 16 (Figure 3.).

\section{Place Figure 3 about here}

\section{Discussion}

Here we report evidence for distinct shifts in the predominant location of slow and fast sleep spindle oscillations during adolescent development. We found evidence for slow sleep spindle duration maxima traveling from central to anterior brain regions, and fast spindle density, amplitude and frequency peaks traveling from central to more posterior brain regions. These findings coincide with reports of an age-related increase in the functional segregation of the precuneus (a major source of fast sleep spindle oscillations in humans) from the frontoparietal region from childhood to adulthood (24). 
In the late maturing frontal and parietal cortical areas, the short-range local connections are dominated by long-range functional networks during development (29). In the course of adolescence, the anterior-posterior connectivity of frontal and parietal lobes increases in the DMN $(30,31)$. Although functional connectivity may be reduced in the DMN in deep sleep stages $(32,33)$, it is preserved to such an extent that oscillatory activity related functional connectivity between anterior and posterior areas corresponds to DMN characteristics during sleep spindling (34).

Former HD-EEG reports, based on sleep slow waves, have shown the anteriorization of sleep state-dependent neural oscillations during human ontogenesis (including the period of adolescence) (25), however, age-related posteriorization of any frequency band has not been shown before. Thus, the late maturation of the frontal lobes is clearly supported by findings of former sleep neurophysiological studies, as well as our present report on slow sleep spindle oscillation, however, the phylogenetically new precuneal region has not yet been targeted by such studies. Although it could have been assumed that the late maturing precuneus is characterized by age-related posteriorization of sleep-state-specific oscillations, indexing the timing of developmental plasticity, such findings have been obscured by the insufficient frequency resolution of former reports. Here we use an individually adjusted frequency analysis of sleep spindle oscillations, with high frequency resolution and 128 channel HD-EEG recording, which is sufficiently sensitive to capture this process. With these more sensitive methods, we have been able to show that individualspecific fast sleep spindle features are unequivocally traveling to more posterior areas during adolescence. Considering the precuneal origin of fast sleep spindles in adult human subjects $(20,21)$, it seems that individualized sleep spindle analysis is a forceful tool to detect the dominant anatomical directions of brain maturation in humans.

Prevailing concepts of complex neural development and brain maturation emphasize the posterior-to-anterior or back-to-front pattern (6-8) as an unequivocal ontogenetic feature, finding its roots in the late maturing frontal lobes. The back-to-front pattern has received substantial support from HD-EEG studies examining the age-related changes in the topography of sleep slow waves (25). However, the above scenario of unidirectional developmental and anatomical route disregards the major hub of the DMN, which is a human-specific and late maturing neural structure of the brain, undergoing significant differentiation during adolescence. Besides slow waves, sleep spindles are the major hallmarks of NREM sleep, known to be involved in neural plasticity with major sources in the precuneus, at least in healthy adult human subjects. Here we provide evidence for a backward move in the anatomical localization of fast type sleep spindles, that is, the predominantly central maxima in density and amplitude at the age of 12 years becomes gradually replaced to the parietal regions by the age of 20 years. Similar changes, albeit at a smaller anatomical distance take place in terms of fast sleep spindle frequency. These findings complete the back-to-front pattern of brain maturation and cohere with the reports of an increased differentiation of the precuneus during adolescence. To the best of our knowledge this is the first report of an age-related front-to-back transfer of the maxima of a neural plasticity-related sleep EEG index. 
Our findings of an age-related decrease in duration and amplitude, as well as an increase in frequency of sleep spindles indicate complex patterns of adolescent brain development. Sleep spindles of unusually high duration have been recorded in infants (35), whereas the decrease in the length of spindle bursts continues during the whole life span (36). Sleep spindle duration has been shown to reflect the level of thalamic inhibition and/or corticothalamic feedback $(37,38)$. That is, our findings might indicate maturation of overall thalamocortical network states reflected in the decreasing length of sleep spindle oscillatory bursts in adolescents. The subtle differences in the developmental trajectories of slow and fast sleep spindle durations suggest the potential divergence in the thalamic and cortical mechanisms involved in generating the two sleep spindle types.

The amplitude of sleep spindles has been shown to reflect the overall level of thalamocortical recruitment and globality of neural sources involved in rhythmogenesis $(39,40)$. The age-related decreases in sleep spindle amplitude reported in the current study and by other researchers (41-43) cohere with the concept of ongoing synaptic pruning during adolescent development, potentially leading to the reduced recruitability of neural generators.

Likewise, the age-related increases in the oscillatory frequency of sleep spindles have been reported by former studies in the field $(41,42,44,45)$. Sleep spindle frequency reflects the modal duration of the single thalamocortical hyperpolarization-rebound sequence embedded in the train of successive spindle cycles. Faster sleep spindles indicate shorter sequence durations, which in turn could be caused by accelerated, more efficient neuronal interactions. We hypothesize that the speeded-up nature of sleep spindles in late adolescence reported herein might reflect ongoing myelination during late adolescence (46). Alternatively, rapid interactions might reflect the above-mentioned changes in network state, characterized by adolescence-related reorganization of the GABAergic system (47).

Apparently, adolescence is a timeframe of significant changes in the EEG spectra, including shifts in sleep spindle characteristics (48-50). Sleep spindles have outstanding relevance from a clinical point of view since adolescence-related psychiatric disorders such as schizophrenia (51), ADHD (52) and depression (53) have all been associated with anomalies in sleep spindles. It has also been suggested that sleep spindle abnormalities are an endophenotype of schizophrenia $(51,54)$. Moreover, these disorders can be characterized with cognitive impairments as well (55-57). It has been demonstrated that sleep spindles and cognitive function are strongly associated $(58,59)$, and as such, sleep spindle abnormalities might provide a key to interpreting cognitive malfunction (17). Further investigating the mechanisms and developmental trajectory of sleep spindles in adolescence could lead to a better understanding and novel treatment of the aforementioned disorders. 
In conclusion, we focused on the late maturation of individually adjusted slow-frontal and fast-parietal sleep spindle features in adolescence by using HD-EEG recordings. Our hypothesis assuming that the two sleep spindle types might index distinct neurodevelopmental processes during the course of adolescent brain maturation and functional specialization has been confirmed by the data. We are convinced that focusing on the bimodal antero-posterior source and frequency distribution of sleep spindle oscillations have taken us closer to a better understanding of the adolescent remodeling of the human brain.

\section{Materials and Methods}

Participants

We recruited 60 adolescent and young adult participants ( 30 females and 30 males, mean age $16.55 \pm \mathrm{SD} 3.70$ ) via social media in three age-groups, with an equal number of females and males in each age group: 12 -year-olds ( $\mathrm{n}=20$, mean age $=12.45 \pm \mathrm{SD} .57$ years), $16-$ year-olds ( $\mathrm{n}=20$, mean age $=15.91 \pm \mathrm{SD} .48$ years), and young adults referred to as 20 -yearolds $(\mathrm{n}=20$, mean age $=21.29 \pm \mathrm{SD} .51$ years). Subjects with an existing neurological condition or sleep disorder were not included in the study. All subjects received a voucher in the value of HUF 20,000 (cca. USD 70.00) for their participation. The study was approved by the Ethical Committee of the Pázmány Péter Catholic University for Psychological Experiments, and participants provided written informed consent.

\section{Procedure}

We performed 128 channel HD-EEG polysomnographic (PSG) sleep recordings of two consecutive nights in the Sleep Laboratory of Pázmány Péter Catholic University Budapest. We requested participants to maintain a regular sleep-wake schedule for 5 nights before recordings, but we did not monitor compliance. We asked participants not to consume any drugs other than contraceptives and coffee, and to refrain from napping on the afternoons of the days of the experiment. Participants with any history of sleep problems or mental disorder, or any neurological or medical condition were excluded. We verified such criteria by personal interviews and questionnaires filled out by the parents or young adult participants themselves. We applied electrodes for electroencephalography (EEG), electromyography (EMG) and electrooculography (EOG). After checking the electrodes for skin contact and impedance levels, all participants went to sleep in the laboratory between $10.00 \mathrm{p} . \mathrm{m}$. and $11.30 \mathrm{p} . \mathrm{m}$. according to their preference. They slept until they awoke spontaneously.

We used a built-in EEG channel-Quick Cap (Compumedics, Australia) with 128 electrodes in three different head sizes to record EEG signals. We roughly evenly spaced the passive $\mathrm{Ag} / \mathrm{AgCl}$ electrodes down to the line T9-Iz-T10. The monopolar channels were referenced to a frontocentrally placed ground electrode. We applied bipolar EOG and EMG channels 
to measure eye movements and muscle tone respectively and placed the EOG channels below and to the left of the left eye ( $1 \mathrm{~cm}$ below the left outer canthus), and above and to the right of the right eye ( $1 \mathrm{~cm}$ above the right outer canthus). We determined the locations of the EMG electrodes in accordance with the recommendations of the American Academy of Sleep Medicine (AASM) (60). We recorded the data using a BQ KIT SD LTM 128 EXPRESS ( 2 X 64 channels in master and slave mode, Micromed, Mogliano Veneto, Italy) recording device, and subsequently visualized, saved and exported them using the System Plus Evolution software (Micromed, Mogliano Veneto, Italy). We recorded all HD-EEG, EOG and EMG data with an effective sampling rate of synchronous $4096 \mathrm{~Hz} /$ channel with 22-bit equivalent resolution, and pre-filtered and amplified them with $40 \mathrm{~dB} /$ decade highand low-pass input filters of $0.15-250 \mathrm{~Hz}$. We applied a digital low pass filter at $231.7 \mathrm{~Hz}$, and downsampled the data to $512 \mathrm{~Hz} / \mathrm{channel}$ by firmware.

From the recorded night-time EEG recordings, the current analysis deals with the second night data as that was free from adaptation effects (first night effect). We scored sleep states of $20 \mathrm{sec}$ epochs of whole night NREM EEG recordings manually by visual inspection according to standardized criteria (61), and revised hypnograms. Then, we removed artefacts on four-second-long epochs and scanned the whole-night recordings four times to overcome the limitations of the capacity of the visual inspector (only 32 channels of the 128 were visually inspected in a run), and finally, we excluded any epoch marked as artefact in any run from further analysis.

We detected individual-specific slow and fast spindles by using the IAM (28). This method is based on the average amplitude spectra of NREM sleep. The frequency criteria of slow and fast sleep spindles were derived from the individual-specific peaks of the spectra between 9 and $16 \mathrm{~Hz}$ based on the inflexion points. We tested the slow and the fast sleep spindle frequencies for frontal and centro-parietal dominance, respectively. We determined the amplitude criteria for slow and fast spindles in individual- and derivation-specific manner by multiplying the number of intra-spindle frequency bins with the mean amplitude spectrum values corresponding to lower and upper frequency limits. Then, we applied a band-pass filter on the EEG for the individual slow and fast sleep spindle frequencies using a fast Fourier transformation filtering method and calculated the precise envelopes of the filtered signals. EEG segments corresponding to the envelopes transcending the amplitude criteria for at least $0.5 \mathrm{~s}$ are considered spindles. A scheme of the spindle detection is provided in Fig. 1 of Ujma et al., 2015 (62). In fact, these segments contribute to the individual- and derivation-specific lower and higher frequency spectral peaks between 9 and $16 \mathrm{~Hz}$. Based on the IAM approach, we determined individual and derivation-specific densities (spindles $\times$ min-1), durations (s), and amplitudes $(\mu \mathrm{V})$ of slow, frontally dominant and fast, centro-parietally dominant sleep spindles.

We determined peak frequency using the center of gravity $(\mathrm{CoG})$ of the individualized spectral peak frequencies in the spindle range $(11-16 \mathrm{~Hz})$. We obtained values of COG by using the method described in the study conducted by Dustman (63) We excluded outliers from the original data set by using Tukey's fences method (64). For testing our hypotheses, we used T-test, post-hoc Fisher LSD test and GLM. 
In order to test our hypotheses, we analyzed the effects of age and gender on the frequency, amplitude, duration and density of slow and fast sleep spindles separately.

We implemented GLM on averaged slow spindle measures of the frontopolar and frontal region (FPF), as well as on averaged fast spindle measures of the centro-parietal (CP) region. We applied two types of models. In the first model, the categorical factors were the age group and the spindle type (slow, fast), and the dependent variable was the spindle parameters of slow and fast spindles. Then, we calculated within effect for spindle type (slow and fast). We computed interaction for Age group, Spindle type and Age group*Spindle type. In the second model, the categorical factors were Sex and Age group; the dependent variables were the spindle parameters of slow and fast spindles. We calculated between effects for Sex, Age group and Sex*Age group interaction. We tested the null hypothesis at the descriptive significance of $p<.05$, then conducted post hoc Fisher's LSD test.

We calculated the distribution of the spindle parameters along the midline derivations (from Fpz to Iz) and determined the maximum position of the distribution for every spindle parameter. We examined the anterior-posterior shifts of the maximum locations as a function of age.

Finally, we analyzed the derivation-specific topographical distribution of spindle parameters and their age-dependent differences. We performed multiple comparisons correction using a modified version of the Rüger area method. We calculated Rüger area global significance as follows: $1 / 3$ of the descriptive significances at $p=.05 / 3=.017$ $\mathrm{AND} / \mathrm{OR}$ half of descriptive significances at $\mathrm{p}=.05 / 2=.025$. In this study, we considered data Rüger area significant under the „AND” condition $(65,66)$.

For the processing of sleep data (sleep state classification, artefact rejection and spindle detection with IAM) we used the FerciosEEGPlus 1.3 program (FerciosEEGPlus, (62)). We used Microsoft Excel for the calculation of $\mathrm{CoG}$ values and Rüger area corrections. We conducted all statistical tests using TIBCO STATISTICA 13.5.0.17 software (TIBCO Software Inc., 2018).

\section{Acknowledgments}

The authors thank $T$. Jáger for generating the figures and tables. We also thank the adolescents and young adults who participated in this project, and Zoltán György who assisted at all the sleep recordings. This research was supported by the Hungarian National Research, Development and Innovation Office grants NK-104481 and K-134370 to I.K., and K-128117 to B.R., and by the Higher Education Institutional Excellence Program of the Ministry of Human Capacities in Hungary, within the framework of the Neurology thematic program of the Semmelweis University. 


\section{References}

1. R. Walker, O. Burger, J. Wagner, C. R. Von Rueden, Evolution of brain size and juvenile periods in primates. J. Hum. Evol. 51, 480-489 (2006).

2. S. R. Leigh, Brain growth, life history, and cognition in primate and human evolution. Am. J. Primatol. 62, 139-164 (2004).

3. A. Gómez-Robles, W. D. Hopkins, S. J. Schapiro, C. C. Sherwood, Relaxed genetic control of cortical organization in human brains compared with chimpanzees. Proc. Natl. Acad. Sci. 112, 14799-14804 (2015).

4. T. Paus, M. Keshavan, J. N. Giedd, Why do many psychiatric disorders emerge during adolescence? Nat. Rev. Neurosci. 9, 947-957 (2008).

5. T. Paus, et al., Maturation of white matter in the human brain: a review of magnetic resonance studies. Brain Res. Bull. 54, 255-266 (2001).

6. J. N. Giedd, Structural Magnetic Resonance Imaging of the Adolescent Brain. Ann. N. Y. Acad. Sci. 1021, 77-85 (2004).

7. E. R. Sowell, et al., Mapping cortical change across the human life span. Nat. Neurosci. 6, 309-315 (2003).

8. R. K. Lenroot, J. N. Giedd, Brain development in children and adolescents: insights from anatomical magnetic resonance imaging. Neurosci. Biobehav. Rev. 30, 718-729 (2006).

9. B. J. Casey, R. M. Jones, T. A. Hare, The Adolescent Brain. Ann. N. Y. Acad. Sci. 1124, 111-126 (2008).

10. L. Steinberg, A Social Neuroscience Perspective on Adolescent Risk-Taking. Dev. Rev. DR 28, 78106 (2008).

11. L. Foulkes, S.-J. Blakemore, Studying individual differences in human adolescent brain development. Nat. Neurosci. 21, 315-323 (2018).

12. F. Váša, et al., Conservative and disruptive modes of adolescent change in human brain functional connectivity. Proc. Natl. Acad. Sci. 117, 3248-3253 (2020).

13. K. J. Whitaker, et al., Adolescence is associated with genomically patterned consolidation of the hubs of the human brain connectome. Proc. Natl. Acad. Sci. U. S. A. 113, 9105-9110 (2016).

14. R. L. Buckner, F. M. Krienen, The evolution of distributed association networks in the human brain. Trends Cogn. Sci. 17, 648-665 (2013).

15. L. Q. Uddin, B. T. T. Yeo, R. N. Spreng, Towards a Universal Taxonomy of Macro-scale Functional Human Brain Networks. Brain Topogr. 32, 926-942 (2019).

16. A. E. Cavanna, M. R. Trimble, The precuneus: a review of its functional anatomy and behavioural correlates. Brain 129, 564-583 (2006).

17. L. M. J. Fernandez, A. Lüthi, Sleep Spindles: Mechanisms and Functions. Physiol. Rev. 100, 805-868 (2020).

18. V. I. Spoormaker, M. Czisch, P. Maquet, L. Jäncke, Large-scale functional brain networks in human non-rapid eye movement sleep: insights from combined electroencephalographic/functional magnetic resonance imaging studies. Philos. Trans. R. Soc. Math. Phys. Eng. Sci. 369, 3708-3729 (2011).

19. D. A. McVea, T. H. Murphy, M. H. Mohajerani, Large Scale Cortical Functional Networks Associated with Slow-Wave and Spindle-Burst-Related Spontaneous Activity. Front. Neural Circuits 10 (2016).

20. P. Anderer, et al., Low-resolution brain electromagnetic tomography revealed simultaneously active frontal and parietal sleep spindle sources in the human cortex. Neuroscience 103, 581-592 (2001).

21. V. Alfonsi, et al., Spatiotemporal Dynamics of Sleep Spindle Sources Across NREM Sleep Cycles. Front. Neurosci. 13 (2019).

22. B. A. Vogt, S. Laureys, "Posterior cingulate, precuneal and retrosplenial cortices: cytology and components of the neural network correlates of consciousness" in Progress in Brain Research, The Boundaries of Consciousness: Neurobiology and Neuropathology., S. Laureys, Ed. (Elsevier, 2005), pp. 205-217.

23. E. Bruner, T. M. Preuss, X. Chen, J. K. Rilling, Evidence for expansion of the precuneus in human evolution. Brain Struct. Funct. 222, 1053-1060 (2017). 
24. R. Li, et al., Developmental Maturation of the Precuneus as a Functional Core of the Default Mode Network. J. Cogn. Neurosci. 31, 1506-1519 (2019).

25. S. Kurth, et al., Mapping of Cortical Activity in the First Two Decades of Life: A High-Density Sleep Electroencephalogram Study. J. Neurosci. 30, 13211-13219 (2010).

26. C. Cajochen, R. Foy, D. J. Dijk, Frontal predominance of a relative increase in sleep delta and theta EEG activity after sleep loss in humans. Sleep Res. Online SRO 2, 65-69 (1999).

27. L. A. Finelli, A. A. Borbély, P. Achermann, Functional topography of the human nonREM sleep electroencephalogram. Eur. J. Neurosci. 13, 2282-2290 (2001).

28. R. Bódizs, J. Körmendi, P. Rigó, A. S. Lázár, The individual adjustment method of sleep spindle analysis: Methodological improvements and roots in the fingerprint paradigm. J. Neurosci. Methods 178, 205-213 (2009).

29. D. A. Fair, et al., Functional Brain Networks Develop from a "Local to Distributed" Organization. PLOS Comput. Biol. 5, e1000381 (2009).

30. J. R. Sato, et al., Age effects on the default mode and control networks in typically developing children. J. Psychiatr. Res. 58, 89-95 (2014).

31. L. E. Sherman, et al., Development of the Default Mode and Central Executive Networks across early adolescence: A longitudinal study. Dev. Cogn. Neurosci. 10, 148-159 (2014).

32. S. G. Horovitz, et al., Decoupling of the brain's default mode network during deep sleep. Proc. Natl. Acad. Sci. 106, 11376-11381 (2009).

33. T. Koike, S. Kan, M. Misaki, S. Miyauchi, Connectivity pattern changes in default-mode network with deep non-REM and REM sleep. Neurosci. Res. 69, 322-330 (2011).

34. Z. Fang, et al., Sleep Spindle-dependent Functional Connectivity Correlates with Cognitive Abilities. J. Cogn. Neurosci. 32, 446-466 (2020).

35. M. J. Spinosa, E. Garzon, Sleep spindles: validated concepts and breakthroughs. J. Epilepsy Clin. Neurophysiol. 13, 179-182 (2007).

36. R. Bódizs, et al., The hemispheric lateralization of sleep spindles in humans. Sleep Spindl. Cortical States 1, 42-54 (2017).

37. M. Bonjean, et al., Corticothalamic Feedback Controls Sleep Spindle Duration In Vivo. J. Neurosci. 31, 9124-9134 (2011).

38. P. Barthó, et al., Ongoing Network State Controls the Length of Sleep Spindles via Inhibitory Activity. Neuron 82, 1367-1379 (2014).

39. E. W. Dempsey, R. S. Morison, The production of rhythmically recurrent cortical potentials after localized thalamic stimulation. Am. J. Physiol.-Leg. Content 135, 293-300 (1941).

40. T. Andrillon, et al., Sleep Spindles in Humans: Insights from Intracranial EEG and Unit Recordings. J. Neurosci. 31, 17821-17834 (2011).

41. I. G. Campbell, I. Feinberg, Maturational Patterns of Sigma Frequency Power Across Childhood and Adolescence: A Longitudinal Study. Sleep 39, 193-201 (2016).

42. S. M. Purcell, et al., Characterizing sleep spindles in 11,630 individuals from the National Sleep Research Resource. Nat. Commun. 8, 15930 (2017).

43. R. S. Nader, C. T. Smith, Correlations between adolescent processing speed and specific spindle frequencies. Front. Hum. Neurosci. 9 (2015).

44. S. Shinomiya, K. Nagata, K. Takahashi, T. Masumura, Development of sleep spindles in young children and adolescents. Clin. EEG Electroencephalogr. 30, 39-43 (1999).

45. A. Goldstone, et al., Sleep spindle characteristics in adolescents. Clin. Neurophysiol. Off. J. Int. Fed. Clin. Neurophysiol. 130, 893-902 (2019).

46. S. Sharma, et al., Maturation of the adolescent brain. Neuropsychiatr. Dis. Treat., 449 (2013).

47. W. Kilb, Development of the GABAergic System from Birth to Adolescence. The Neuroscientist 18, 613-630 (2012).

48. A. Nicolas, D. Petit, S. Rompré, J. Montplaisir, Sleep spindle characteristics in healthy subjects of different age groups. Clin. Neurophysiol. 112, 521-527 (2001).

49. L. Tarokh, E. Van Reen, M. LeBourgeois, R. Seifer, M. A. Carskadon, Sleep EEG Provides Evidence that Cortical Changes Persist into Late Adolescence. Sleep 34, 1385-1393 (2011).

50. L. Tarokh, M. A. Carskadon, Developmental Changes in the Human Sleep EEG During Early Adolescence. Sleep 33, 801-809 (2010). 
51. D. S. Manoach, J. Q. Pan, S. M. Purcell, R. Stickgold, Reduced Sleep Spindles in Schizophrenia: A Treatable Endophenotype That Links Risk Genes to Impaired Cognition? Biol. Psychiatry 80, 599608 (2016).

52. I. Merikanto, et al., ADHD symptoms are associated with decreased activity of fast sleep spindles and poorer procedural overnight learning during adolescence. Neurobiol. Learn. Mem. 157, 106-113 (2019).

53. D. T. Plante, et al., Topographic and sex-related differences in sleep spindles in major depressive disorder: A high-density EEG investigation. J. Affect. Disord. 146, 120-125 (2013).

54. I. Merikanto, et al., Genetic risk factors for schizophrenia associate with sleep spindle activity in healthy adolescents. J. Sleep Res. 28 (2019).

55. R. Göder, et al., Impairment of sleep-related memory consolidation in schizophrenia: relevance of sleep spindles? Sleep Med. 16, 564-569 (2015).

56. M. Loyer Carbonneau, M. Demers, M. Bigras, M.-C. Guay, Meta-Analysis of Sex Differences in ADHD Symptoms and Associated Cognitive Deficits. J. Atten. Disord., 108705472092373 (2020).

57. P. L. Rock, J. P. Roiser, W. J. Riedel, A. D. Blackwell, Cognitive impairment in depression: a systematic review and meta-analysis. Psychol. Med. 44, 2029-2040 (2014).

58. M. Hahn, et al., Developmental changes of sleep spindles and their impact on sleep-dependent memory consolidation and general cognitive abilities: A longitudinal approach. Dev. Sci. 22, e12706 (2019).

59. C. M. Reynolds, M. A. Short, M. Gradisar, Sleep spindles and cognitive performance across adolescence: A meta-analytic review. J. Adolesc. 66, 55-70 (2018).

60. C. Iber, S. Ancoli-Israel, A. L. Chesson Jr., S. F. Quan, The AASM Manual for Scoring of Sleep and Associated Events Rules, Terminology and Technical Specifications (American Academy of Sleep Medicine, 2007) (May 16, 2021).

61. AASM Scoring Manual - American Academy of Sleep Medicine. Am. Acad. Sleep Med. - Assoc. Sleep Clin. Res. (May 14, 2021).

62. P. P. Ujma, P. Sándor, S. Szakadát, F. Gombos, R. Bódizs, Sleep spindles and intelligence in early childhood-developmental and trait-dependent aspects. Dev. Psychol. 52, 2118-2129 (2016).

63. R. E. Dustman, D. E. Shearer, R. Y. Emmerson, Life-span changes in EEG spectral amplitude, amplitude variability and mean frequency. Clin. Neurophysiol. 110, 1399-1409 (1999).

64. J. W. Tukey, Exploratory Data Analysis, Addison-Wesley Series in Behvioral Science: Quantitative Methods (Addison-Wesley Publishing Company, 1977) (March 31, 2021).

65. B. Rüger, Das maximale signifikanzniveau des Tests: "LehneHo ab, wennk untern gegebenen tests zur ablehnung führen." Metrika 25, 171-178 (1978).

66. K. Abt, Descriptive data analysis: a concept between confirmatory and exploratory data analysis. Methods Inf. Med. 26, 77-88 (1987). 


\section{Figures}

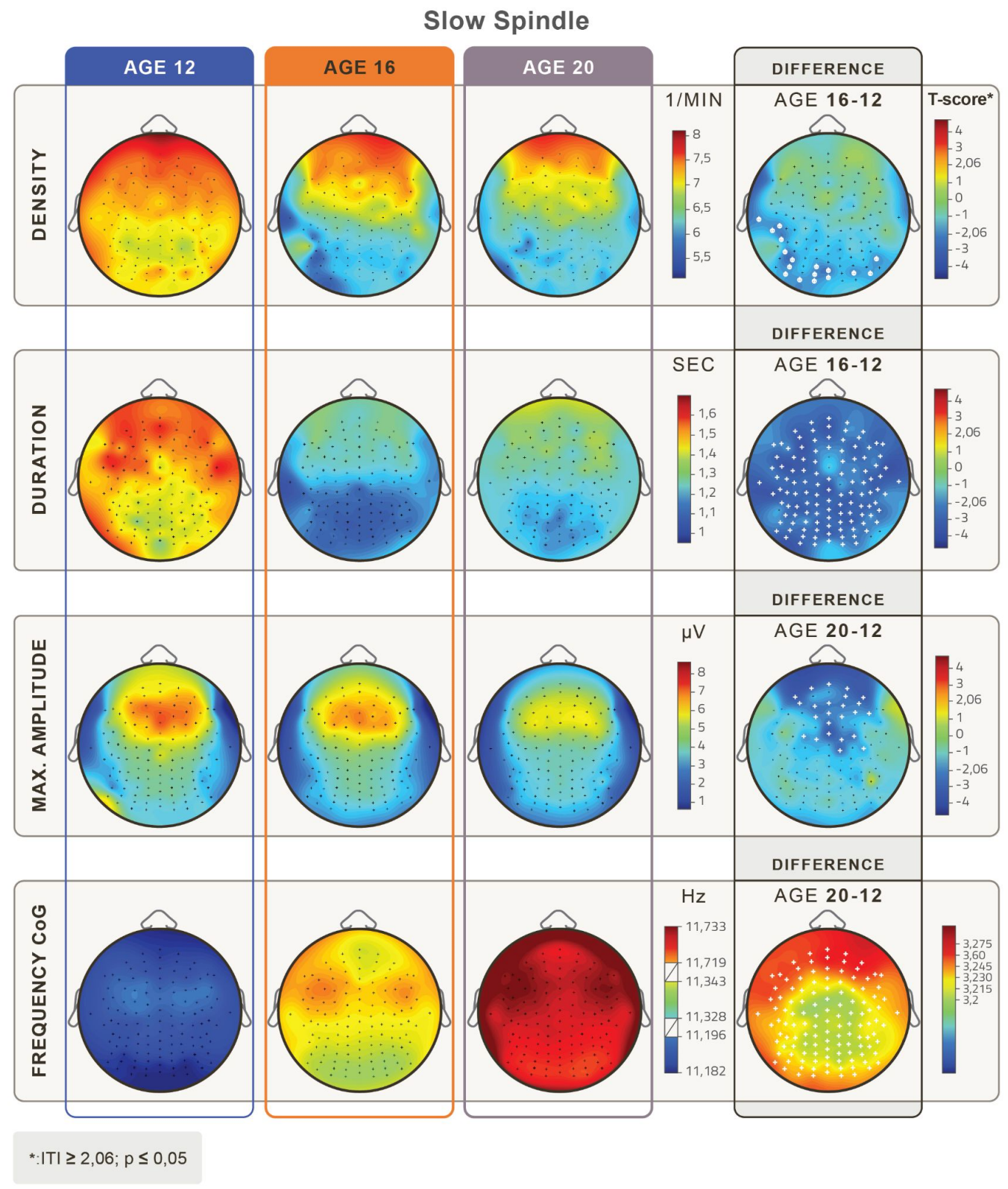

Figure 1. Topographical distribution of mean slow sleep spindle parameters in the age groups of 12,16 and 20 years of age. Rows depict age-related changes in slow sleep spindle density (1/min), duration (s), maximum amplitude $(\mu \mathrm{V})$, and frequency based on the center of gravity $(\mathrm{CoG})(\mathrm{Hz})$, respectively. Relevant age-group differences are depicted in the 4 th column. White crosses depict Rüger area significant differences and white circles depict significant but not Rüger area significant differences. Age related decrease of density is significant with an occipital and left temporal focus. There was a significant overall decrease in duration and frontal-frontopolar decrease in maximum amplitude. Frequency increases at all derivations with a frontal focus from the younger to the older age groups. (Level of significance: $p<.05$, T-score values are matched with the colorbar, $\mathrm{T} \geq|2.06| \mathrm{p}<0.05$, at $\mathrm{T} \geq|2.787| \mathrm{p}<0.01$ and at $\mathrm{T} \geq|3.725| \mathrm{p}<0.001$.) 


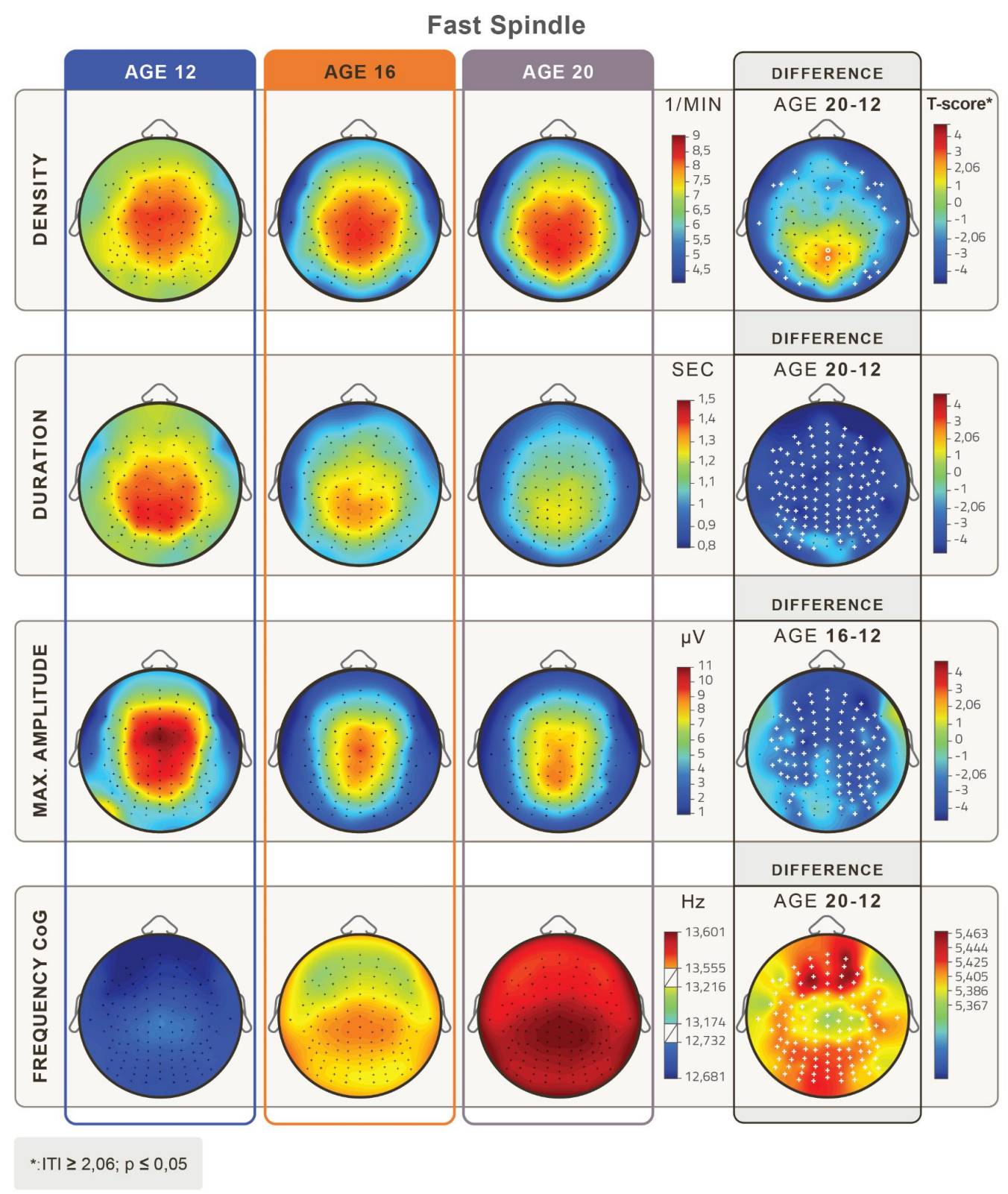

Figure 2. Topographical distribution of mean fast sleep spindle parameters in the age groups of 12, 16 and 20 years. Rows depict age-related changes in slow sleep spindle density ( $1 / \mathrm{min})$, duration (s), maximum amplitude $(\mu \mathrm{V})$, and frequency based on the center of gravity $(\mathrm{CoG})(\mathrm{Hz})$, respectively. Relevant age-group differences are depicted in the 4th column. White crosses depict Rüger area significant differences and white circles depict significant but not Rüger area significant differences. Fast spindle density increased significantly, but not Rüger significantly centro-parietally between 12 and 20 years of age and Rüger significantly decreased at the perimeter of the scalp. There is a significant overall decrease in duration and a frontal decrease in maximum amplitude. Frequency significantly increased at all derivations with frontocentral maxima from the younger to the older age group. (Level of significance: $\mathrm{p}<.05$, T-score values are matched with the colorbar, $\mathrm{T} \geq|2.06| \mathrm{p}<0.05$, at $\mathrm{T} \geq|2.787| \mathrm{p}<0.01$ and at $\mathrm{T} \geq|3.725| \mathrm{p}<0.001$.) 


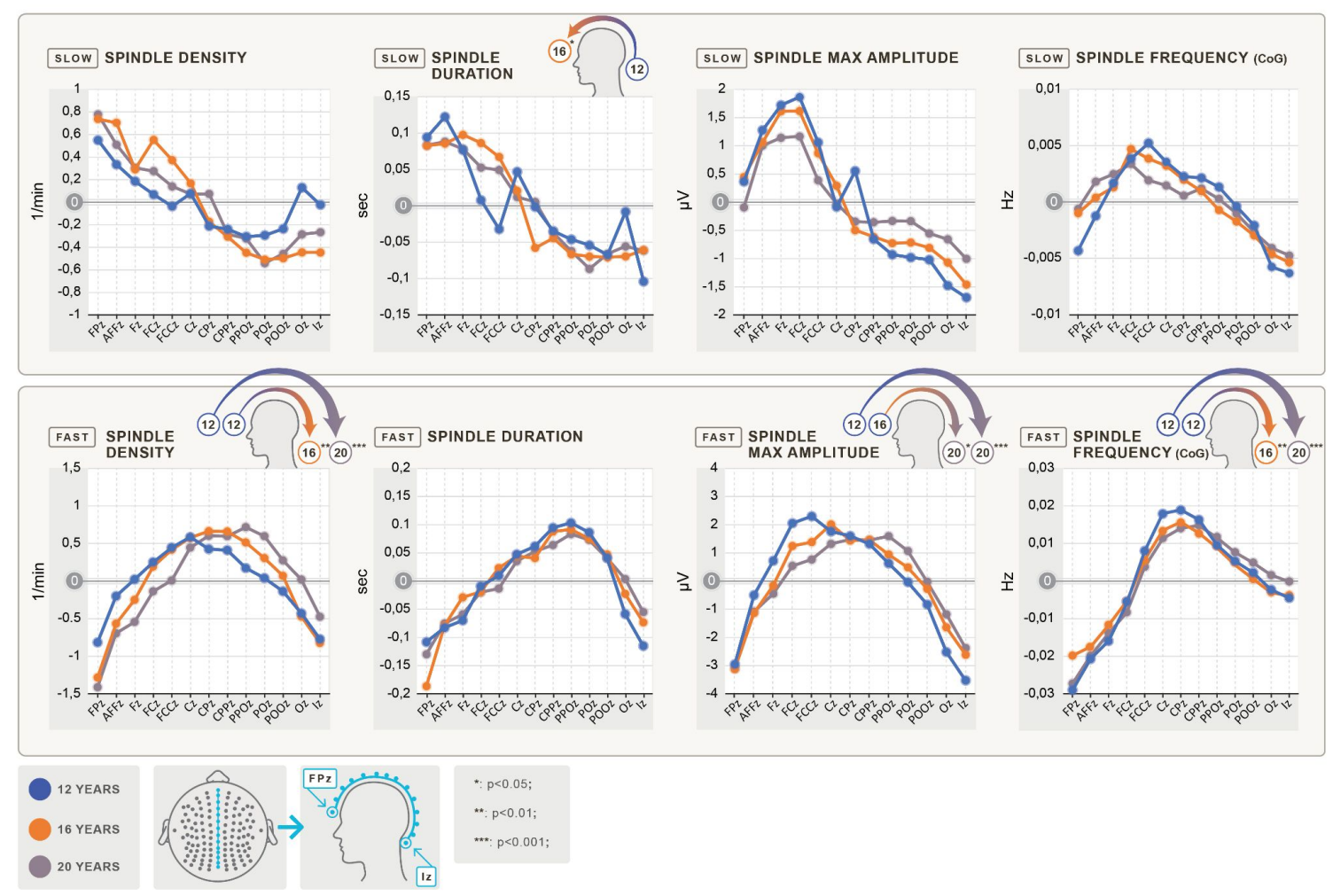

Figure 3. Age-related antero-posterior shifts in sleep spindle parameters. The spindle parameter values (y-axis) for the midline electrodes (Fpz to Iz on the x-axis, from left to right from frontal to occipital) are plotted by age groups. As the differences are significant but small (differences due to spatial position are much smaller than differences between age groups), the y-values display the difference from the mean value of the midline electrodes of the specific age group. The upper row depicts slow spindle parameters, while the lower row depicts fast spindle parameters. The different columns show different spindle parameters such as density $(1 / \mathrm{min})$, duration $(\mathrm{sec})$, maximum amplitude $(\mu \mathrm{V})$ and frequency $(\mathrm{Hz})$ based on the center of gravity $(\mathrm{CoG})$, respectively. The small head figures above the graphs indicate significant differences and the direction of the difference in the positions of the maximum values between the specific age groups. Blue color indicates $12-$, orange color 16- and grey color 20 -year-old subjects. Slow spindle parameters tended to shift toward anterior, whereas fast sleep spindle measures toward posterior regions with age. The shift of the maximum values of slow spindle duration towards frontal regions was statistically significant. In terms of fast spindle density, maximum amplitude and frequency, the shift of the maximum values from central to centro-parietal regions was statistically significant. 


\section{Supplementary Information}

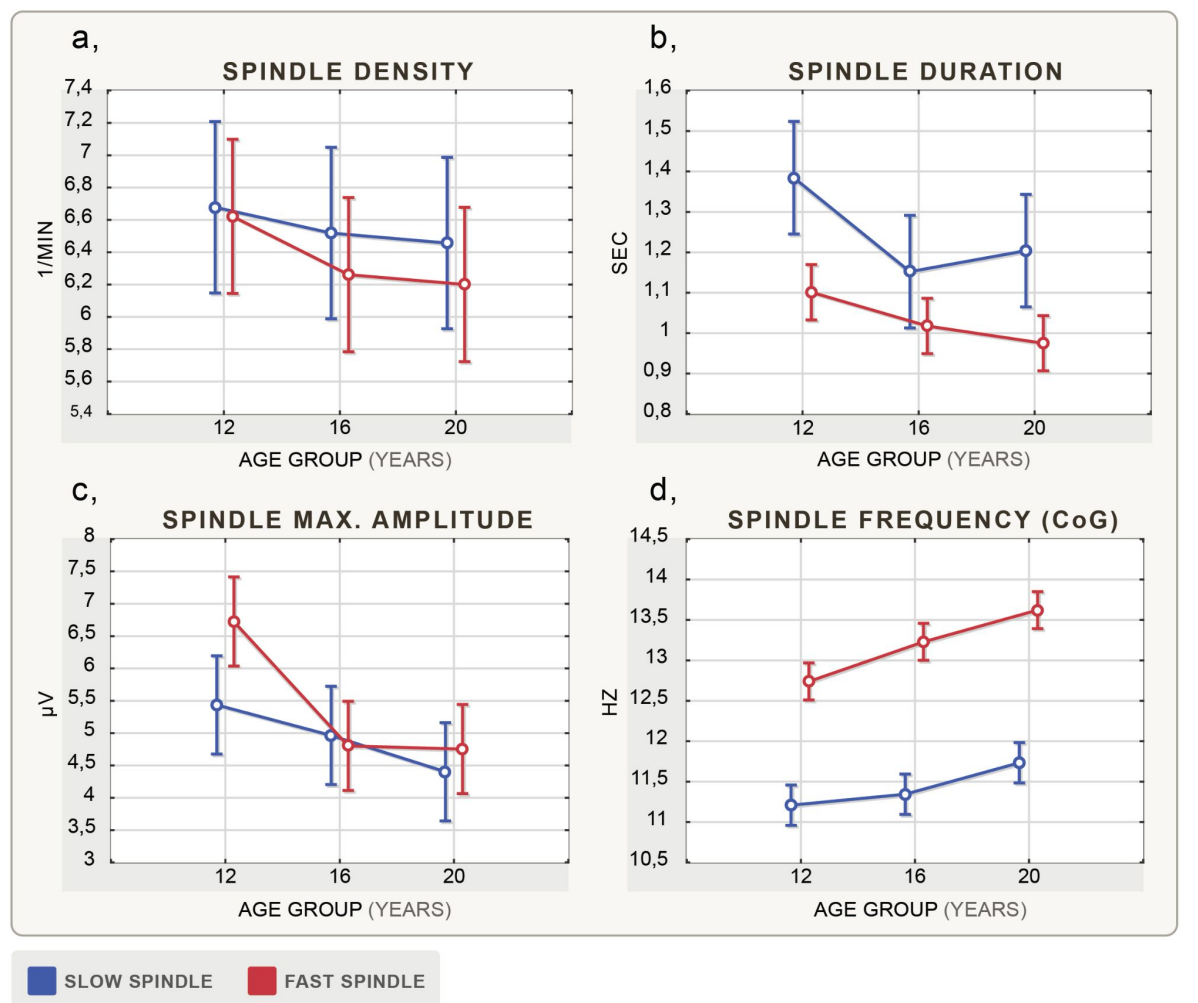

Supplementary Information Figure 1. Age-related changes of NREM sleep spindle parameters. General Linear Models (GLMs) were implemented with categorical factors: Age groups (12 years, 16 years, 20 years) and the dependent variables a, Spindle density $(1 / \mathrm{min})$, b, Spindle duration $(\mathrm{sec}), \mathbf{c}$, Spindle maximum amplitude $(\mu \mathrm{V})$, d, Spindle frequency $(\mathrm{Hz})$ based on center of gravity $(\mathrm{CoG})$ of individually adjusted spindle bands. Vertical bars denote 0,95 confidence intervals, the central point of bars represent average values for each age group. Blue color denotes slow spindle, red color denotes fast spindle. ANOVA revealed no significant main effect of age on spindle density $(\mathrm{F}=.599 ; \mathrm{df}=2,57 ; \mathrm{p}=.552)$, however the main effect of age was significant in terms of duration, $(\mathrm{F}=4.217 ; \mathrm{df}=2,57 ; \mathrm{p}=.019)$, maximum amplitude $(\mathrm{F}=6.498 ; \mathrm{df}=2,57$; $\mathrm{p}=.002)$ and frequency $(\mathrm{F}=10.47 ; \mathrm{df}=2,57 ; \mathrm{p}<.001)$. Post hoc Fisher's LSD test demonstrated significant agedependent decrease for slow spindle duration between the age groups 12 and $16(\mathrm{p}=.003)$ as well as 12 and $20(\mathrm{p}=.022)$, but not between 16 and 20 years (SI Figure 1b). Similarly, slow spindle amplitude decreased significantly from age 12 to 20 years $(\mathrm{p}=.046)$, while the decrease of fast spindle amplitude was significant between ages 12 and $16(p<.001)$ as well as 12 and $20(p<.001)$. Spindle frequency increased significantly in both spindle types between all age groups except for slow spindles between the age groups of 12 and 16 years, i.e. slow spindle frequency increased between 12 and 20 years $(\mathrm{p}=.002)$ as well as 16 and 20 years $(\mathrm{p}=.002)$, while fast spindle frequency increased between 12 and 20 years $(\mathrm{p}<.001), 12$ and 16 years $(\mathrm{p}=.005)$ as well as 16 and 20 years $(\mathrm{p}=.024)$. As revealed by ANOVA, the difference of density between the two spindle types was not significant $(\mathrm{F}=1.818 ; \mathrm{df}=2,57 ; \mathrm{p}=.183)$, however slow spindles displayed significantly longer duration than fast spindles $(\mathrm{F}=32.073 ; \mathrm{df}=2,57 ; \mathrm{p}<.001)$. This difference was prominent $(\mathrm{p}<.001)$ in all age groups. Fast spindles showed significantly higher maximum amplitude at age 12 than slow spindles $(\mathrm{p}<.001)$, but the difference disappeared with ageing. The main distinctive feature of the slow and fast spindles was in frequency, where fast spindles displayed significantly higher frequency in all age groups at a significance of $\mathrm{p}<.001$. 
Sex differencies in spindle frequency (CoG)

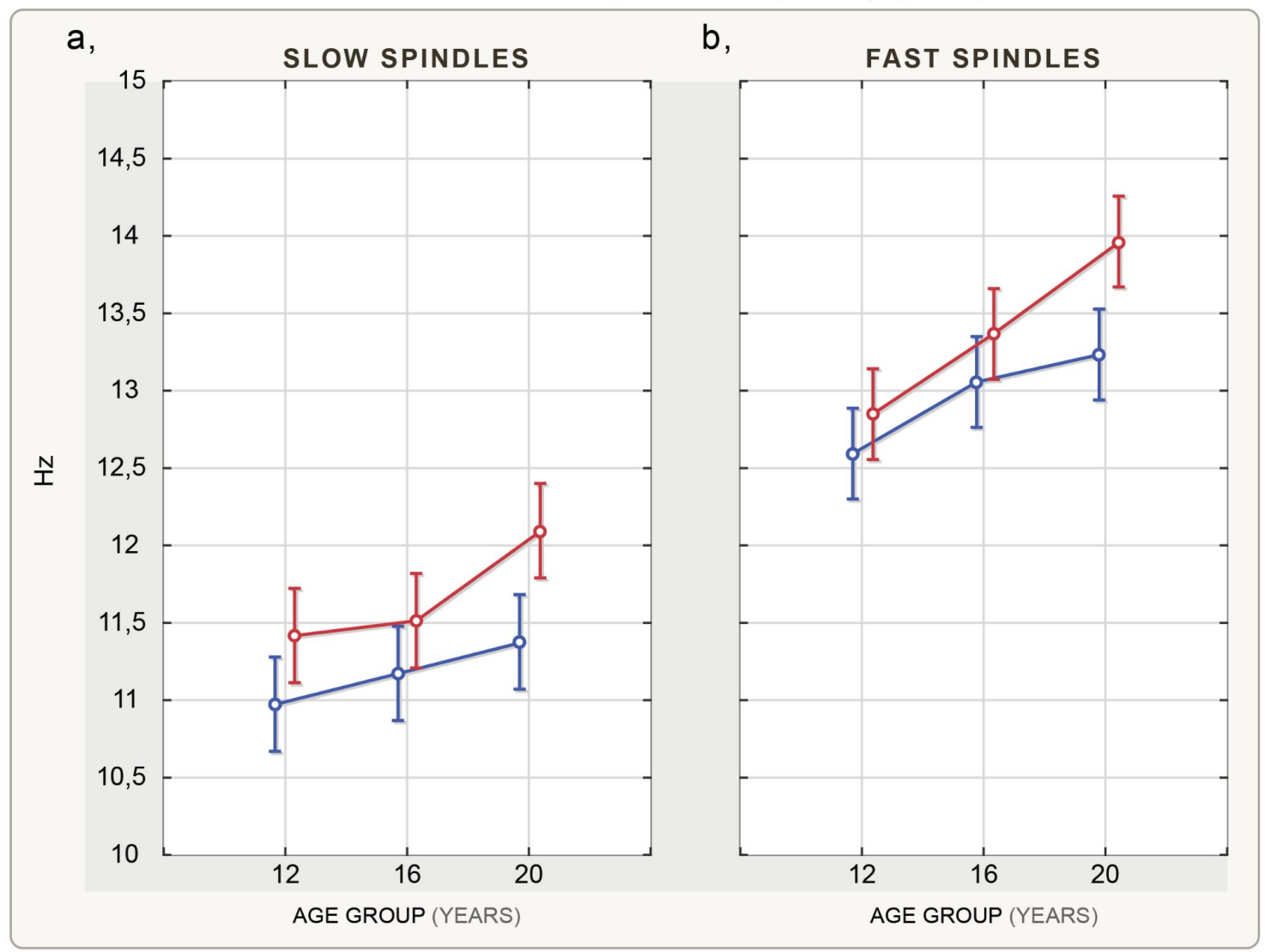

MALE

FEMALE

Supplementary Information Figure 2. Sex and age-related changes (12 years, 16 years, 20 years) of NREM sleep spindle frequency based on the center of gravity $(\mathrm{CoG})$ of individually adjusted spindle bands $(\mathrm{Hz})$ is depicted on the left side of the figure for slow spindles, and on the right side for fast spindles. Vertical bars denote 0,95 confidence intervals, the central point of bars represent average values for each age group. Color codes blue: male, red: female. We found no evidence for the influence of sex on the development of NREM sleep spindle parameters except for frequency, where we found a significant main effect of sex $(F=18.11 ; d f=2,57 ; p=<.001)$. a, Girls have a significantly higher frequency of slow spindles than boys in the age groups of 12 and 20 years $\left(M_{12}=11.192, p=.04 ; M_{20}=11.73, p=.001\right)$. $\mathbf{b}$, sex difference in fast sleep spindles was significant only in the age group of 20 years $\left(M_{20}=13.595, p<.001\right)$. Girls between 16 and 20 years exhibited faster increase in frequency than boys. 